\title{
Secretory Leukocyte Protease Inhibitor in Oral Potentially Malignant Disorders and Oral Squamous Cell Carcinoma
}

\begin{abstract}
Objectives: Secretory leukocyte protease inhibitor (SLPI) is a protein found in body fluids and implicated as a prognostic factor in many cancers. There is limited research regarding serum and salivary SLPI in oral potentially malignant disorders (OPMDs) and oral squamous cell carcinoma (OSCC). Materials and Methods: A total of 90 participants were included in the study; of which 30 formed the control group, 30 included participants with OPMD, and 30 included participants with OSCC. Serum and salivary levels of SLPI were estimated using enzyme-linked immunosorbent assay. Results: Serum levels of SLPI showed progressive increase in potentially malignant disorders and SCC patients when compared with controls, while salivary levels were increased in both OPMD and OSCC groups as compared to controls. However, the difference between groups was not statistically significant $(P<0.05)$. Conclusion: The above results suggest that increase in SLPI levels in the serum may have a role in indicating progression of OPMD to OSCC.
\end{abstract}

Keywords: Oral potentially malignant disorders, oral squamous cell carcinoma, saliva, Secretory leukocyte protease inhibitor, serum

\section{Introduction}

Oral cancer is the $15^{\text {th }}$ most prevalent cancer worldwide with a $50 \%$ survival rate despite recent advances in surgery, chemotherapy and radiotherapy. ${ }^{[1]}$ More than $95 \%$ of oral cancers are squamous cell carcinomas (SCCs). ${ }^{[2]}$ The high incidence of oral cancer in India is attributed to the habits of tobacco chewing and smoking. These carcinogens interact with the oral mucosa to cause premalignant and malignant changes. Most malignancies of the oral cavity arise from preexisting premalignant lesions. ${ }^{[1]}$ With a view to prevention of oral potentially malignant disorders (OPMD) developing into oral SCCs (OSCCs), various studies have tried to identify biomarkers in the serum and saliva.

Secretory leukocyte protease inhibitor (SLPI) was first isolated from secretions of patients with chronic obstructive pulmonary disease and cystic fibrosis and was thereby considered a major anti elastase inhibitor. SLPI is produced by neutrophils, macrophages, beta-cells of

\footnotetext{
This is an open access journal, and articles are distributed under the terms of the Creative Commons Attribution-NonCommercial-ShareAlike 4.0 License, which allows others to remix, tweak, and build upon the work non-commercially, as long as appropriate credit is given and the new creations are licensed under the identical terms.
}

For reprints contact: reprints@medknow.com pancreatic islets, epithelial cells investing the renal tubules, acinar cells of parotid and submandibular glands, acinar cells of submucosal glands, and epithelial cells lining mucous membranes of respiratory and alimentary tracts. SLPI protects the tissues by inhibiting proteases such as cathepsin G, elastase, and trypsin from neutrophils; chymotrypsin; and trypsin from pancreatic acinar cells and chymase and tryptase from mast cells. The main action is inhibition of neutrophil elastase which protects epithelial surfaces from damage. ${ }^{[3]}$ Initially studied for its role in the prevention of HIV transmission in saliva, SLPI has recently been recognized as a potential diagnostic and prognostic marker in the head-and-neck cancer. ${ }^{[4]}$ It is found in most secretions of the body and mainly in saliva. ${ }^{[5]}$ Previous research on SLPI has used brush biopsy specimens in OPMD and OSCC. ${ }^{[6]}$ This report demonstrated a significant decrease in SLPI in participants with oral precancer and cancer with the levels decreasing progressively from oral precancer to cancer. A recent study has evaluated archived salivary samples of patients who went on to develop OSCC and found that high salivary SLPI in smokers

How to cite this article: Ajila V, Babu S, Shetty V,
Hegde S, Shenoy S. Secretory leukocyte protease
inhibitor in oral potentially malignant disorders and
oral squamous cell carcinoma. Indian J Med Paediatr
Oncol 2019;40:491-5.

How to cite this article: Ajila V, Babu S, Shetty V, Hegde S, Shenoy S. Secretory leukocyte protease oral squamous cell carcinoma. Indian J Med Paediatr Oncol 2019;40:491-5

\section{Vidya Ajila1, Subhas Babu', Veena Shetty², Shruthi Hegde ${ }^{1}$, Shilpa Shenoy ${ }^{3}$}

${ }^{1}$ Department of Oral Medicine and Radiology, A. B. Shetty Memorial Institute of Dental Sciences, Nitte Deemed to be University, ${ }^{2}$ Department of Microbiology, K. S. Hegde Medical Academy, Nitte Deemed to be University, ${ }^{3}$ Central Research Laboratory, K. S. Hegde Medical Academy, Nitte Deemed to be University, Mangalore, Karnataka, India

Submitted: 05-Apr-2018 Revised: 18-May-2018 Accepted: 21-Jun-2018 Published: 17-Feb-2020

Address for correspondence: Dr. Vidya Ajila, Department of Oral Medicine and Radiology, A. B. Shetty Memorial Institute of Dental Sciences, Nitte Deemed to be University, Mangalore, Karnataka, India. E-mail: ajila_v@yahoo.com

Access this article online

Website: www.ijmpo.org

DOI: 10.4103/ijmpo.ijmpo_75_18 Quick Response Code:

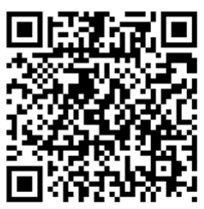


was associated with increased risk of the head-and-neck SCCs (HNSCC) ${ }^{[5]}$ However, studies which have evaluated serum and salivary SLPI in OPMD and OSCC are lacking.

The present study aims to evaluate the role of serum and salivary SLPI in progression of OPMD into OSCC.

\section{Materials and Methods}

A total of 90 participants reporting to the outpatient department of our institute were included in the study. They were divided into three groups of 30 participants each: Group I included participants diagnosed with OPMD, Group II included participants diagnosed with OSCC, and Group III formed the control group. Participants with any underlying systemic disease were excluded from the study. Institutional Ethics Committee Clearance and written informed consent were obtained from each study participant. Demographic data were acquired from the participants and were followed by an examination of the oral cavity. Venous blood was withdrawn from the antecubital vein and serum obtained. Whole saliva was collected by asking the participants to allow saliva to collect in the mouth and then spit into sterile containers every minute for $5 \mathrm{~min}$. Saliva and serum were stored at $-20^{\circ}$ until analysis.

Serum and salivary SLPI was determined using ELISA (Hycult Laboratories, Netherlands). The results obtained were subjected to statistical analysis.

\section{Statistical analysis}

Kruskal-Wallis test was used to compare the three groups. Intergroup comparison was done using MannWhitney U-test. Correlation analysis was performed using Spearman's correlation test.

\section{Results}

Of the 30 participants with OPMD (study Group 1), 26 were male and 4 were female. The third, fourth, and fifth decades all had equal number of cases. Oral submucous fibrosis (OSMF) was the most common lesion in this group followed by leukoplakia and erosive lichen planus [Figure 1]. Gutkha chewing was the most common etiologic agent in OSMF, while smoking was associated with leukoplakia. Study Group II (OSCC) consisted of 24 males and 6 females. Maximum cases were seen in the $7^{\text {th }}$ decade followed by the $6^{\text {th }}$ decade. The most common site was the buccal mucosa followed by the tongue and the alveologingival sulcus [Figure 2]. Betel-quid chewing (BLAT) was the most common associated etiological factor [Figure 3].

\section{Serum Secretory leukocyte protease inhibitor}

The mean level of SLPI in the control, OPMD, and OSCC groups were 26.90, 44.78, and $105.56 \mathrm{pg} / \mathrm{ml}$, respectively. The difference between the groups was statistically nonsignificant [Figure 4].

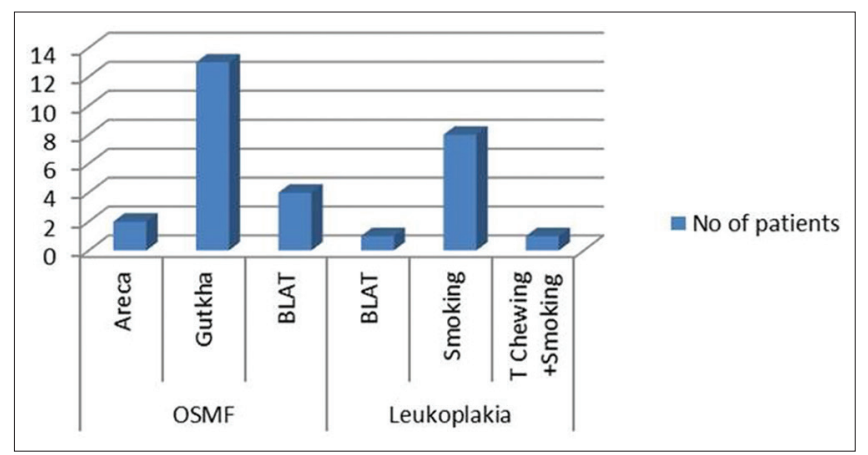

Figure 1: Graph showing the habits associated with oral potentially malignant disorders. Gutkha chewing was the most common etiologic agent in oral submucous fibrosis while smoking was associated with leukoplakia

\section{Salivary Secretory leukocyte protease inhibitor}

The mean level of SLPI in the control, OPMD, and OSCC groups were 80.28, 103.60, and $92.76 \mathrm{pg} / \mathrm{ml}$, respectively. The difference between the groups was statistically nonsignificant [Figure 5].

\section{Discussion}

SLPI is an $11.7 \mathrm{kDa}$ highly basic nonglycosylated protein. SLPI contributes to host mucosal defense of oral, nasopharyngeal, genital, and respiratory tissues. Salivary SLPI was initially studied for its anti-HIV activity. ${ }^{[7]}$ Studies have shown a protective function for SLPI against human papillomavirus (HPV) infection of the oral cavity. ${ }^{[8]}$ It has a major role in protecting tissues against the harmful effects of inflammation. ${ }^{[3]}$ It also has bactericidal and fungicidal properties. ${ }^{[3]}$ Even though the anti-HIV property of SLPI is well established, there appears to be no role for SLPI in other retroviral infections. ${ }^{[3]}$

Recently, SLPI is being investigated for its role in a variety of cancers such as ovarian, breast, colorectal, pancreatic, and oral cancer. ${ }^{[6,9-13]}$ It has been implicated in cancer progression and metastasis. ${ }^{[13]}$ Cordes et al..$^{[14]}$ demonstrated that lower SLPI was associated with metastatic HNSCC. Devoogdt et $a l^{[15]}$ studied the role of SLPI in lung tumor progression and found that SLPI had definite promalignant activity. The authors concluded that this property of SLPI was due to its protease inhibiting activity rather than its property of increasing cell proliferation. ${ }^{[15]}$ Zhang et al. ${ }^{[13]}$ proved that elevated SLPI in pancreatic adenocarcinoma was associated with cell proliferation and growth of tumor. ${ }^{[13]}$ Decreased SLPI is believed to promote spread of the tumor by allowing degradation of tissues by proteases produced by tumor cells. ${ }^{[8]}$

Quabius et al. ${ }^{[16]}$ found that decreased SLPI was associated with HPV-induced OSCC, while smoking-induced OSCC had higher levels of SLPI and absence of HPV positivity. Quabius et al..$^{[16]}$ proposed that SLPI protects against HPV-induced OSCC in nonsmokers. SLPI increases in response to smoking and thus high levels are seen in smokers. ${ }^{[8]}$ Pierce Campbell et al. found that salivary SLPI levels were inversely correlated with age. ${ }^{[17]}$ 


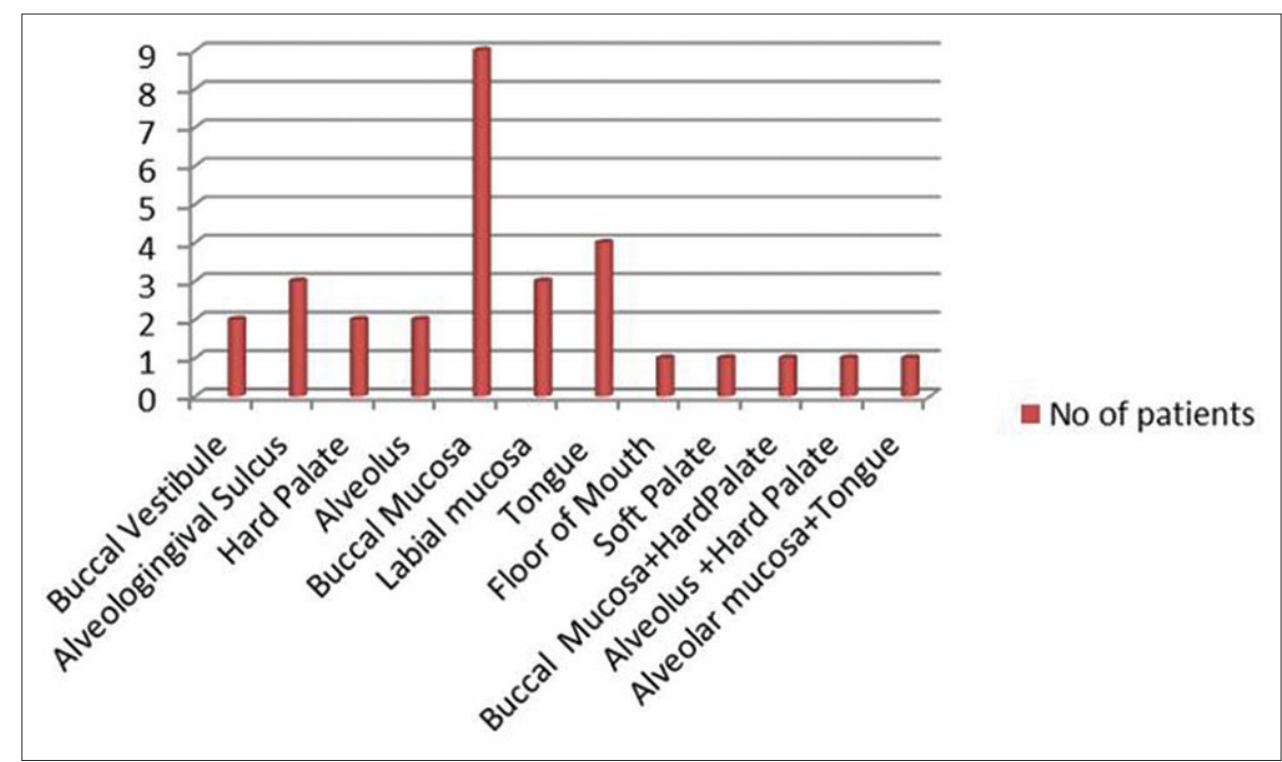

Figure 2: Graph showing the site distribution of oral squamous cell carcinoma. The most common site was the buccal mucosa followed by the tongue



Figure 3: Graph showing the habits associated with oral squamous cell carcinoma. Betel-quid chewing was the most common associated etiological factor

The present study evaluated the serum and salivary SLPI in participants diagnosed with OPMD and OSCC to determine its role in the progression of precancer to cancer.

Male preponderance was seen in both OPMD and OSCC groups which is similar to previous literature reports. ${ }^{[18,19]}$

OPMD were diagnosed from the third to fifth decade of life, whereas OSCC occurred predominantly in the $7^{\text {th }}$ decade. This is in conformity with previous studies which have stated that OSCC occurs later due to accumulation of age-related mutagenic and epigenetic effects as also because most OSCC is believed to arise from pre-existing OPMD. ${ }^{[20]}$

OPMDs are a group of oral conditions which include leukoplakia, erythroplakia, erosive lichen planus, and oral submucous fibrosis. The buccal mucosa was the most common site of OSCC in the present study. Previous studies conducted in India have reported similar

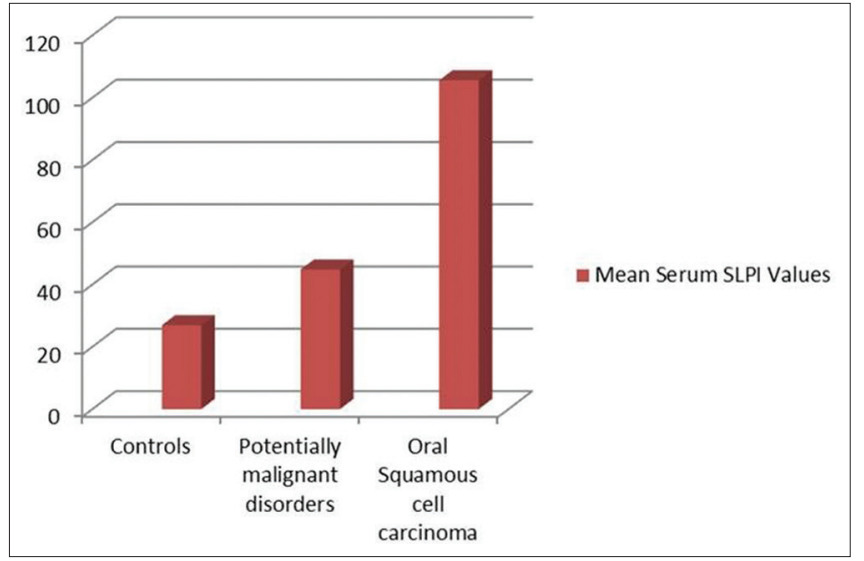

Figure 4: Progressive increase in mean levels of serum secretory leukocyte protease inhibitor in oral potentially malignant disorders and oral squamous cell carcinoma as compared to controls

findings. ${ }^{[20]}$ Smoking was the etiological factor in most cases with leukoplakia, while gutkha chewing was associated with oral submucous fibrosis. Betel quid, a form of chewing tobacco which is composed of areca nut, lime, tobacco, and betel leaf, was the etiological agent in most cases of OSCC. According to the World Health Organization, 34\% of the population uses beedis, 31\% uses cigarettes, $19 \%$ uses smokeless tobacco, $9 \%$ uses hookah, and $7 \%$ use other types of tobacco, while the Cancer Patients Aid Association of India found that cigarettes accounted for $20 \%$, beedis $40 \%$, and chewable tobacco accounted for $40 \%$ of tobacco use in India. ${ }^{[21]}$

A mass spectrometry-based quantitative shotgun proteomics study of oral brush biopsy specimens found that SLPI was progressively decreased in patients with OPMD and OSCC as compared to healthy controls. ${ }^{[6]}$ This decrease was correlated with the development of OPMD and OSCC. They further suggested that treatment with SLPI could have 




Figure 5: Increased mean levels of salivary secretory leukocyte protease inhibitor in oral potentially malignant disorders and oral squamous cell carcinoma as compared to controls

preventive effects. ${ }^{[6]}$ The present study compared the serum and salivary levels of SLPI in patients with OPMD, OSCC, and healthy controls.

The present study found that serum levels of SLPI increased progressively from controls to OPMD patients to OSCC patients. The difference between the groups was not statistically significant. We were unable to correlate these results with literature reports since previous studies on serum SLPI levels in OPMD and OSCC are not available.

Salivary levels were higher in OPMD and OSCC as compared to controls. However, the difference between the groups was not significant. Wen et al. ${ }^{[22]}$ found that SLPI was inversely correlated with tumor invasion in the head-and-neck OSCC and has implications for prognosis and treatment planning. However, in ovarian cancers, SLPI was found to be upregulated. ${ }^{[11,22]}$ Pierce Campbell et al. ${ }^{[5]}$ evaluated salivary SLPI and found that higher SLPI levels among smokers showed a higher risk for development of OSCC.

Decreased SLPI levels have been associated previously with a higher risk of tissue invasion. The present study showed higher levels of SLPI in OPMD and OSCC. These findings could probably be explained by the anti-inflammatory properties of SLPI which led to increased levels in saliva and serum. Although the results in the present study were not statistically significant, there was progressive increase in serum SLPI from controls to OPMD to OSCC indicating that serum SLPI could have a role in evaluating the progression of OPMD to OSCC.

The major limitation of the study is the sample size. Our study was more in the nature of a pilot study, and therefore, studies with larger sample size are required before definitive conclusions can be drawn.

\section{Conclusion}

SLPI is believed to have a role in tumor progression. It also has potential roles in prognosis and treatment planning in OSCC. Studies on SLPI in different types of tumors have provided conflicting results. Salivary and serum levels of SLPI have not been evaluated so far. Our study aimed to determine the role of SLPI as a prognostic factor in the progression of OPMD to OSCC. The present study showed increase in serum SLPI in cases with OPMD and OSCC as compared to controls. Salivary levels of SLPI were also increased in OPMD and OSCC as compared to controls. Our results may reflect the anti-inflammatory properties of SLPI. These preliminary results appear to indicate that increasing levels of SLPI in the serum may indicate progression to OSCC. However, since the sample size of the present study was small, large-scale studies are required before any definitive results.

\section{Financial support and sponsorship}

The study was funded by NITTE University Research Grant.

\section{Conflicts of interest}

There are no conflicts of interest.

\section{References}

1. Shah FD, Begum R, Vajaria BN, Patel KR, Patel JB, Shukla SN, et al. A review on salivary genomics and proteomics biomarkers in oral cancer. Indian J Clin Biochem 2011;26:326-34.

2. Rivera C, Venegas B. Histological and molecular aspects of oral squamous cell carcinoma (Review). Oncol Lett 2014;8:7-11.

3. Doumas S, Kolokotronis A, Stefanopoulos P. Anti-inflammatory and antimicrobial roles of secretory leukocyte protease inhibitor. Infect Immun 2005;73:1271-4.

4. Pushpanshu K, Sathawane RS, Kaushik R. Estimation and comparison of salivary secretory leukocyte protease inhibitor in human immunodeficiency virus patients and healthy individuals. Indian J Palliat Care 2014;20:26-30.

5. Pierce Campbell CM, Giuliano AR, Torres BN, O'Keefe MT, Ingles DJ, Anderson RL, et al. Salivary secretory leukocyte protease inhibitor (SLPI) and head and neck cancer: The cancer prevention study II nutrition cohort. Oral Oncol 2016;55:1-5.

6. Yang Y, Rhodus NL, Ondrey FG, Wuertz BR, Chen X, Zhu Y, et al. Quantitative proteomic analysis of oral brush biopsies identifies secretory leukocyte protease inhibitor as a promising, mechanism-based oral cancer biomarker. PLoS One 2014;9:e95389.

7. Lin AL, Johnson DA, Stephan KT, Yeh CK. Salivary secretory leukocyte protease inhibitor increases in HIV infection. J Oral Pathol Med 2004;33:410-6.

8. Hoffmann M, Quabius ES, Tribius S, Hebebrand L, Görögh T, Halec G, et al. Human papillomavirus infection in head and neck cancer: The role of the secretory leukocyte protease inhibitor. Oncol Rep 2013;29:1962-8.

9. Wang G, Lim DS, Choi BD, Park JJ, Jeong SJ, Kim JS, et al. Effect of secretory leukocyte protease inhibitor on migration and invasion of human KB oral carcinoma cells. Animal Cells Syst 2011;15:139-46.

10. Liu G, Yang J, Zhao Y, Wang Z, Xing B, Wang L, et al. Expression of secretory leukocyte protease inhibitor detected by immunohistochemistry correlating with prognosis and metastasis in colorectal cancer. World J Surg Oncol 2014;12:369.

11. Simpkins FA, Devoogdt NM, Rasool N, Tchabo NE, Alejandro EU, 
Kamrava MM, et al. The alarm anti-protease, secretory leukocyte protease inhibitor, is a proliferation and survival factor for ovarian cancer cells. Carcinogenesis 2008;29:466-72.

12. Sayers KT, Brooks AD, Sayers TJ, Chertov O. Increased secretory leukocyte protease inhibitor (SLPI) production by highly metastatic mouse breast cancer cells. PLoS One 2014;9:e104223.

13. Zhang W, Yao JL, Dong SC, Hou FQ, Shi HP. SLPI knockdown induced pancreatic ductal adenocarcinoma cells proliferation and invasion. Cancer Cell Int 2015;15:37.

14. Cordes $\mathrm{C}$, Häsler R, Werner $\mathrm{C}$, Görögh $\mathrm{T}$, Röcken $\mathrm{C}$, Hebebrand L, et al. The level of secretory leukocyte protease inhibitor is decreased in metastatic head and neck squamous cell carcinoma. Int J Oncol 2011;39:185-91.

15. Devoogdt N, Hassanzadeh Ghassabeh G, Zhang J, Brys L, De Baetselier P, Revets H, et al. Secretory leukocyte protease inhibitor promotes the tumorigenic and metastatic potential of cancer cells. Proc Natl Acad Sci U S A 2003;100:5778-82.

16. Quabius ES, Möller P, Haag J, Pfannenschmidt S, Hedderich J, Görögh $\mathrm{T}$, et al. The role of the antileukoprotease SLPI in smoking-induced human papillomavirus-independent head and neck squamous cell carcinomas. Int J Cancer 2014;134:1323-34.
17. Pierce Campbell CM, Guan W, Sprung R, Koomen JM, O'Keefe MT, Ingles DJ, et al. Quantification of secretory leukocyte protease inhibitor (SLPI) in oral gargle specimens collected using mouthwash. J Immunol Methods 2013;400-401:117-21.

18. Salian V, Dinakar C, Shetty P, Ajila V. Etiological trends in oral squamous cell carcinoma: A retrospective institutional study. Cancer Transl Med 2016;2:33-6.

19. Mathur PT, Dayal PK, Pai K. Correlation of clinical patterns of oral squamous cell carcinoma with age, site, sex and habits. J Indian Acad Oral Med Radiol 2011;23:81-5.

20. Feller L, Lemmer J. Oral squamous cell carcinoma: Epidemiology, clinical presentation and treatment. J Cancer Ther 2012;3:263-8.

21. Balakrishnan C, Aswath N. Estimation of serum, salivary immunoglobulin G, immunoglobulin A levels and total protein, hemoglobin in smokeless tobacco chewers and oral submucous fibrosis patients. Contemp Clin Dent 2015;6:S157-62.

22. Wen J, Nikitakis NG, Chaisuparat R, Greenwell-Wild T, Gliozzi M, Jin W, et al. Secretory leukocyte protease inhibitor (SLPI) expression and tumor invasion in oral squamous cell carcinoma. Am J Pathol 2011;178:2866-78. 